\title{
Tolerance of clinical uncertainty by geriatric residents: a qualitative study
}

\author{
Marianne B. van lersel ${ }^{1}$ (D) Esther Brantjes ${ }^{1} \cdot$ Marieke de Visser $^{2} \cdot$ Natasja Looman $^{3} \cdot$ Ellen Bazelmans $^{4}$. \\ Dieneke van Asselt ${ }^{1}$
}

Received: 18 February 2019 / Accepted: 29 April 2019 / Published online: 8 May 2019

(c) The Author(s) 2019

\section{Key summary points}

Aim To investigate qualitatively how residents cope with clinical uncertainty while caring for their complex geriatric patients. Findings Residents experience clinical uncertainty and judge it positive as well as negative. Despite the impact on their feelings, behavior and well-being, they do not discuss it with others.

Message Clinical uncertainty is an inherent part of caring for complex geriatric patients and influences the well-being of residents, but training in tolerance of clinical uncertainty is lacking.

\begin{abstract}
Context With the growing complexity in health care, clinical uncertainty increases, even more so in geriatrics. Intolerance of clinical uncertainty can result in stress, burnout and additional costs. This makes tolerance of clinical uncertainty a highly relevant skill to learn. This study investigated how residents cope with clinical uncertainty and explored options to improve their tolerance of it. Methods We interviewed nine residents from the geriatric department of a university medical center and analyzed the interviews conform template analysis using the 'integrative model of uncertainty tolerance'.

Results All residents experienced clinical uncertainty regularly and emphasized it was a relevant topic. Residents described clinical uncertainty as both negative and positive, explaining it was difficult to deal with and could lead to stress, but it also kept them focused, challenged them and stimulated learning. While most of the reported topics fitted in the theoretical model, the model did not reflect the dynamics of clinical uncertainty and lacked its consequences outside the workplace. Residents mainly responded to clinical uncertainty by asking supervisors and peers to double-check their decisions concerning a patient. Residents indicated that they barely discussed their own emotions, cognitions or learning processes with peers or their supervisors. They would welcome the incorporation of clinical uncertainty as standard theme in patient supervision and educational meetings.

Conclusion Clinical uncertainty is not a problem of an insecure, failing resident, but an inherent part of caring for complex geriatric patients. Residents deserve to be trained in tolerance of clinical uncertainty to improve their well-being and care for geriatric patients.
\end{abstract}

Keywords Clinical uncertainty $\cdot$ Medical education $\cdot$ Resident $\cdot$ Complexity

Marianne B. van Iersel

marianne.vaniersel@ radboudumc.nl

1 Department of Geriatric Medicine, Radboud University, Medical Center, Internal code 925, PO Box 9101, 6500 HB Nijmegen, The Netherlands

2 Department for Research in Learning and Education, Radboudumc Health Academy, Nijmegen, The Netherlands

3 Department of Primary Care, Radboud University Medical Center, Nijmegen, The Netherlands

4 Department of Medical Psychology, Radboud University Medical Center, Nijmegen, The Netherlands

\section{Introduction}

Clinical uncertainty (CU) has always been an inherent part of medical practice, arising from diagnostic problems, ambiguities of treatment and unpredictability of patient response. Nowadays, clinical uncertainty is even more prominent in the fast growing group of frail older patients with atypical symptoms, multimorbidity, cognitive impairment and functional decline. Physicians treating these patients often have to make decisions based on limited knowledge, because their patients are underrepresented in clinical trials and single 
disease guidelines do not suit these complex patients [1,2]. In other words, clinical uncertainty can be increased at four domains and all four apply to frail older patients [3]. First, the atypical symptoms and cognitive impairment increase uncertainty in the patient's story. Second, the scarce scientific evidence causes uncertainty about what best to do diagnostically and therapeutically. Third, in case of multimorbidity lack or conflicting advices from the relevant disease guidelines poses questions about what best to do. Last, with multiple problems how best to collaborate with all involved health-care workers can be uncertain too.

Part of being a physician is to recognize and cope with $\mathrm{CU}$, but the level of tolerance differs between physicians [4]. Tolerance of $\mathrm{CU}$ is associated with professional wellbeing: Physicians who report lower tolerance of uncertainty more often experience reactions such as stress and avoidance when placed in ambiguous and uncertain situations [5-8] and may be at increased risk of burnout [9]. CU also influences direct patient care: Physicians with a low tolerance for uncertainty are more likely to use extensive testing to reduce their uncertainty about diagnoses, which paradoxically increases the risk of patient harm and comes with additional costs $[10,11]$.

As medical trainers we aim to coach our residents on coping with $\mathrm{CU}$, but we found little footing in literature. Most previous studies had medical students and consultants as subject group and all only used standard questionnaires. [12].

Therefore this study's primary aim is to investigate qualitatively how residents experience and respond to $\mathrm{CU}$ in complex situations in geriatrics. The secondary aim is to explore the educational needs of residents to improve their tolerance of CU.

\section{Methods}

We used semi-structured interviews followed by template analysis [13] to gain understanding of the meaning of CU in residents. We report the methods according to the COREQ criteria [14].
The study population consisted of residents working at the department of geriatric medicine of the Radboud University Medical Center. The interviewer (E Brantjes) introduced herself and this study to the residents through a short pitch during an educational meeting. Afterward, she approached the residents by email with an invitation to participate, which they all agreed to. Table 1 shows the demographic characteristics of the participants.

The interviewer did not know the participants, but could relate to their situation because of her previous internship in geriatrics. During the study, she conducted and analyzed all interviews. She acquired her interview techniques during her medical training and received additional training in qualitative methods and analysis. She was part of the research group that encompasses two geriatricians, one resident in geriatrics, one general practitioner, two psychologists and one educationalist.

\section{Data collection}

In total, the interviewer conducted nine semi-structured, face to face interviews in July and August 2018 near the participants' workplace. She conducted the interviews in Dutch during one-on-one conversations that lasted 45-60 min, also taking field notes. To structure the interviews, she used an interview guide (examples in Table 2), composed after consulting literature and discussions in the research group. The 'integrative model of uncertainty tolerance' of Hillen [15] formed the core template of the interview and analysis. This model encompasses the perception of clinical uncertainty, reactions in the cognitive, emotional and behavioral domains and their influencing factors. For the secondary aim of the study, the interviewer asked open questions about previous education in $\mathrm{CU}$ and wishes for future training in this subject. The interviewer pilot tested the interview guide to adjust it wherever needed and to reflect on her interview competence. This process was repeated throughout the interviewing process if new subjects or questions arose.

All interviews were audio recorded and transcribed verbatim. The interviews were conducted and analyzed sequentially. After seven interviews, no more new information
Table 1 Demographic characteristics of participants

\begin{tabular}{lllll}
\hline & Age & Gender & $\begin{array}{l}\text { Years of special- } \\
\text { ist training } \\
\text { Mean (range) }\end{array}$ & $\begin{array}{l}\text { Years as a physician } \\
\text { Mean (range) }\end{array}$ \\
\hline $\begin{array}{l}\text { Resident in training for } \\
\text { Geriatric medicine }\end{array}$ & $28-32$ & 1 Male, 5 female & $3.7(1-5)$ & $4.5(2-6)$ \\
Nursing home medicine & 30 & 1 Male & 2 & 5 \\
Internal medicine & 33 & 1 Female & 6 & 7 \\
General practitioner & 28 & 1 Female & 2 & 3 \\
Mean (range) & $30(28-33)$ & & $3.3(1-6)$ & $4.7(2-7)$ \\
\hline
\end{tabular}


emerged and saturation was reached. We performed two more interviews for verification and discussed saturation within the research group.

\section{Data analysis}

The interviewer anonymized, transcribed, read and reread interviews to obtain an overall understanding of the written text. She coded the data by open coding followed by axial coding, using Atlas.ti 8 software [14] for analyses, coding and memo writing. We had chosen Nigel King's Template analysis as method to analyze coded data [13]. We used the 'integrative model of uncertainty tolerance' of Hillen [15] as our template and open questions about future education in CU. We adjusted it throughout the analytical process. In this process, the interviewer engaged in one-on-one discussions with different members of the research team, studied relevant literature and revisited interviews regularly. The interviewer kept a logbook to summarize and reflect on the evolving analytical process, which she shared and discussed with the other researchers. The final template contained a hierarchical list of themes, categories and codes.

To increase validity, we performed a member check and peer debriefing by reporting and discussing the preliminary analysis with participants and new residents from the geriatric department. We incorporated their comments in the analysis.

Because the analytical process was in Dutch, our native language, we held a discussion with the research group and consulted a native English speaker in case of doubt to refine the English translations used in this report.

\section{Ethical considerations}

The ethical committee of the Radboud University Medical Centre waivered approval for our study. All participants received written information about the study and signed informed consent that they had understood the information and we could use the data for research purposes.

\section{Results}

All participants perceived CU regularly and emphasized that $\mathrm{CU}$ is a relevant topic for them. We identified six themes concerning CU: stimulus, perception, moderators, appraisal/ response, secondary consequences of and need for focus on CU. These themes reflect and partially expand the existing elements of the 'integrative model of uncertainty tolerance' [15]. The experienced significance of each theme varied between participants. We will discuss the themes consecutively in the following paragraphs.

\section{Stimulus}

Different aspects in the clinical situation (complexity, ambiguity and probability) can cause CU. We asked participants in which situation they perceived CU. The majority of participants told us that either the diagnostic and therapeutic path (complexity) or the unpredictability of future outcomes (probability) caused feelings of CU. Some of the participants mentioned a combination of both aspects. The diagnostic and therapeutic path can cause CU because for every problem several possible solutions exist, with minimal scientific underpinning and often no clear best option at the start.

'To me it is the choice between all sorts of possible treatments and doing nothing, and the uncertainty that it creates, that you do not know how it will turn-out.' (P1)

Adding to $\mathrm{CU}$ is the limited value of guidelines to the participants (ambiguity). Some felt that guidelines reduce $\mathrm{CU}$, while others considered that they often do not apply to the individual patient or that advices contradict each other. One participant said:

'We have frail patients with a lot of co-morbidities to which standard guidelines often do not apply'. (P2)
Table 2 Example of the interview guide

\begin{tabular}{ll}
\hline Domain & Question (example) \\
\hline Current situation & Please describe a work situation in which you experienced CU \\
Behavior & What did you do to reduce uncertainty? \\
Cognitive & What were your thoughts and considerations in this situation? \\
Emotional & Can you tell me how you emotionally responded to this situation? \\
Afterward & What did you do to cope with this situation? \\
Certainty & Which factors help you to judge a situation as certain? \\
Training & Is clinical uncertainty addressed in your training and, if so, how? \\
\hline
\end{tabular}




\section{Perception}

Participants need to recognize signs to realize that they are dealing with CU. To most participants signs of CU were worrying and to doubt decisions. One participant put it as:

'When continuously thinking about it. At a certain point I should be done worrying'. (P6)

Not all participants were immediately aware of their CU and perception of $\mathrm{CU}$ could take time.

Signs of peers coping with CU might be mumbling behind the computer or asking questions looking for confirmation. It turned out that mutual awareness and exchange about the perception of CU between residents is limited. For example, one participant said:

'I always think of others, 'oh they know it all, they are so good they never doubt'”. (P7)

\section{Moderators}

The perception of CU is moderated by individual, situational and social characteristics. The most prominent moderators are social characteristics like role models and the learning environment. Role models, both positive and negative, are mainly supervisors and peers. The learning environment includes time and physical space to meet each other, but also the threshold residents perceive to discuss CU. In case of no time, space or a high threshold, they looked for other options to discuss $\mathrm{CU}$.

'It really depends on the connection you have with people. Do you feel comfortable in the situation you're in? If not, I think you will talk about it at home or with friends'. (P8)

Residents associated work experience and personality traits like perfectionism with the perception of CU. The majority of participants agreed with the statement that experience as a physician reduces CU. On the opposite, one participant mentioned:

'If I look back on the first years of my residency, I perceived little uncertainty at the time. Maybe I was still naif and ignorant, didn't know all the things you can miss or do wrong'. (P7)

A recurrent theme in the interviews was the wish of participants 'to do the right thing' and the experienced responsibility to do so. Some participants wanted to satisfy the patient and his family, while others considered that they did the right thing if they had done their utmost.

A few participants also mentioned external factors like colleagues and peculiarities of patients and their families.

\section{Appraisal/response}

A participants' response after perceiving CU can be cognitive, emotional and behavioral. A common cognition among participants was that $\mathrm{CU}$ was part of the deal. It comes with being a doctor. Even though most participants perceived $\mathrm{CU}$ as difficult, in all cases, the participants agreed that it was also something positive. One participant said:

'I think it is important to perceive CU to check for yourself whether the things you do are correct and if they are right for the patient.' (P4)

The perceived difficulty of $\mathrm{CU}$ resulted in emotions like sadness, worry, irritation and disappointment. When perceiving $\mathrm{CU}$, participants often discussed these patients with their supervisor or peers. These discussions mainly encompassed uncertainties about the medical situation of that patient to gather knowledge and confirmation. In these discussions, residents only implicitly pointed to their feeling of uncertainty using the word 'difficult'. One of the participants stated:

'I ask the supervisor, do you agree with me? So you

try, if you have doubts, to ask confirmation of someone

else about the choices you had made.' (P6)

Whenever their supervisor did not fulfill this need for conformation, residents turned to third parties like secretaries or partners at home. Surprisingly, only a minority of residents double-checked with the patient to confirm if they were on the right track or not. Another often reported coping strategy was distraction such as driving home, music and sports.

\section{Secondary consequences}

A reported theme, not present in the theoretical model, was the influence of $\mathrm{CU}$ on private life. To several participants this influence was mainly working late, but to some it influenced their psychosocial well-being. One of the participants said:

'I was not happy at my workplace and because of that not at home. I was quite often gloomy, worrying a lot, not sleeping well, having nightmares.'(P7)

\section{Need for focus on CU}

While the majority of the participants agreed that they would appreciate more awareness concerning $\mathrm{CU}$, a minority mentioned that they do not share that opinion. One of the participants commented: 
'I do not need much extra guidance. [...] just discussing this is enough sometimes'. (P3)

\section{Medical education}

Last, we asked participants what they need in their training to improve their coping with CU. They mainly wished more time and space to discuss and share cognitions and emotions of $\mathrm{CU}$ with their supervisors and their peers.

'I think it can contribute, especially when you share emotions, not only my emotions but to hear from your supervisor what kind of emotions he has. Maybe he experiences stress as well because he is uncertain about a decision and how he copes with that.' (P2)

Additionally, participants suggested there should be moments for residents to share experiences and emotions in a small group with an independent supervisor.

\section{Discussion}

This study determined six themes to feed our understanding of CU in complex situations in residents. These themes are stimuli, perception, moderators, appraisal/response, secondary consequences of and need for focus on CU. All participants perceived CU regularly and emphasized that it is a topic of relevance to them. However, the importance of the themes varied between participants. The residents wished that $\mathrm{CU}$ will become part of regular medical training because of the influence of $\mathrm{CU}$ on their well-being.

\section{Tolerance of $\mathrm{CU}$ in residents caring for frail older patients}

This is the first study about CU in residents instead of physicians in general, consultants or medical students. Residents form a specific group because they are less experienced than a medical specialist and have many new responsibilities. In residents, $\mathrm{CU}$ is associated with stress as well [7, 9], but the link with extensive testing in this study was less clear than previously described $[10,11]$. The participating residents did not spontaneously mention the effect of CU on ordering additional tests, but when asked mentioned that they did think that $\mathrm{CU}$ is a trigger for them to do so. An explanation for this may be that participants are unaware that the root of their actions is CU. Second, appropriate ordering of tests is an explicit topic of supervision, because geriatricians know that tests can harm patients, cost extra money and do not necessarily help in decision making.

We had chosen the themes from the 'integrative model of uncertainty tolerance' to reflect on and expand the existing theory on how residents tolerate CU [15]. Our study confirms that overall the model is applicable in medical practice. However, the residents described the aspects of $\mathrm{CU}$ as dynamic instead of static as described by the theoretical model. For example, responses to and secondary consequences of $\mathrm{CU}$ in one situation influence the perception of $\mathrm{CU}$ in a subsequent situation. Extra checks may cause residents to work late, making them more tired the next day, consequently influencing their perception or response to CU. Also, the model suggests that responses to $\mathrm{CU}$ can either be positive or negative. Our study showed that responses cannot easily be distinguished in these two contrasting categories. For example, the response to $\mathrm{CU}$, mainly double-checking, can be positive until this becomes too time consuming or inefficient. A third limitation of the model is that it does not take consequences of $\mathrm{CU}$ on private life into account. The addition of this aspect would do justice to the growing attention to decrease stress and prevent burnout.

This qualitative research method with semi-structured interviews facilitated the detailed examination of the meaning of CU. In-depth interviews are not restricted to specific questions and can be guided by the researcher, but the participant is free to state whatever he thinks is important. This enabled us to get a broad impression of $\mathrm{CU}$ in residents. As in all face-to-face interviews, participants may tend to give socially desirable answers. Because some responses were the opposite of what was expected, we believe we had created a safe interview environment in which residents felt free to speak honestly.

We conducted all interviews in Dutch, but reported them in English. To limit the possibility of results being lost in translation, we discussed translations with a native English speaker to find the best suited words, concepts and sentences. This study was also limited by the absence of a second coder. To minimize possible bias, the researcher took reflective notes, revisited data numerous times and engaged in one-on-one discussions with different members of the team.

\section{Incorporation in medical training}

A key component to improve the tolerance of $\mathrm{CU}$ in residents is the time and possibility to discuss and share cognitions and emotions of $\mathrm{CU}$ with their supervisors or peers. This can shift the focus from feeling uncertain as a personal failure to $\mathrm{CU}$ as integral part of caring for complex geriatric patients. A workshop with a short general presentation about $\mathrm{CU}$ and thereafter a guided discussion in small groups seems in pilots a good start. However, a single workshop requires a transfer of knowledge from classroom to clinical practice and presumably will not be enough to change attitude and improve tolerance of $\mathrm{CU}$ in daily practice [16]. Options to learn how to tolerate $\mathrm{CU}$ at the workplace include addressing 
it in clinical rounds and patient supervision, and discussion of the topic in mentor and intervision meetings. Furthermore, consultants have to be role models in their coping with $\mathrm{CU}$ and probably learn to better tolerate $\mathrm{CU}$ themselves too and share their experiences with residents.

Several questions remain unresolved. This study was carried out at the geriatric department of a university hospital. We presume that $\mathrm{CU}$ is present in residents from other departments, but that the perception of it may be different because of the variation in individual characteristics of residents, patient population, learning environment and specialty-specific language. In our preliminary and unpublished data, pediatric residents had equal scores on the Gerrity scale of tolerating clinical uncertainty as geriatric residents. From discussions with residents in microbiology, neurology and internal medicine, we learned that almost all residents experience clinical uncertainty. Every specialty, however, uses different words to describe it. For example, surgeons talk about 're-evaluating and adapting a plan' when addressing clinical uncertainty, or 'complex anatomy' that takes additional time during surgery [17]. To develop a better picture of $\mathrm{CU}$, additional studies will be needed that include residents of other departments.

In conclusion, despite $\mathrm{CU}$ being omnipresent in the complex health care for geriatric patients with much impact on the well-being of residents, residents lack training in how to tolerate CU. We strongly recommend the field to incorporate strategies to cope with $\mathrm{CU}$ in the workplace learning of residents.

Author contributions MI: study concept and design, acquisition of subjects, analysis and interpretation of data and preparation of manuscript. EB: study concept and design, acquisition of subjects, analysis and interpretation of data, and preparation of manuscript. MV: study concept and design, interpretation of data, and preparation of manuscript. NL: study concept and design, interpretation of data, and preparation of manuscript. EB: study concept and design, interpretation of data, and preparation of manuscript. DA: study concept and design, acquisition of subjects and/or data, analysis and interpretation of data, and preparation of manuscript.

\section{Compliance with ethical standards}

Conflict of interest The authors declare that they have no conflict of interest.

Ethical approval The ethical committee of the Radboud University Medical Centre waivered approval for our study.

Informed consent All participants received written information about the study and signed informed consent that they had understood the information and we could use the data for research purposes.

Open Access This article is distributed under the terms of the Creative Commons Attribution 4.0 International License (http://creat ivecommons.org/licenses/by/4.0/), which permits unrestricted use, distribution, and reproduction in any medium, provided you give appropriate credit to the original author(s) and the source, provide a link to the Creative Commons license, and indicate if changes were made.

\section{References}

1. Boyd CM, Darer J, Boult C et al (2005) Clinical practice guidelines and quality of care for older patients with multiple comorbid diseases: implications for pay for performance. JAMA 294:716-724

2. Plsek PE, Greenhalgh T (2001) Complexity science: the challenge of complexity in health care. BMJ 323:625-628

3. Greenhalgh T, Howick J, Maskrey N (2014) Evidence based medicine renaissance G. Evidence based medicine: a movement in crisis? BMJ 348:g3725

4. Simpkin AL, Schwartzstein RM (2016) Tolerating uncertaintythe next medical revolution? N Engl J Med 375:1713-1715

5. Bovier PA, Perneger TV (2007) Stress from uncertainty from graduation to retirement-a population-based study of Swiss physicians. J Gen Intern Med 22:632-638

6. Iannello P, Mottini A, Tirelli S, Riva S, Antonietti A (2017) Ambiguity and uncertainty tolerance, need for cognition, and their association with stress. A study among Italian practicing physicians. Med Educ Online 22:1270009

7. Lally J, Cantillon P (2014) Uncertainty and ambiguity and their association with psychological distress in medical students. Acad Psychiatry 38:339-344

8. Simpkin AL, Khan A, West DC et al (2018) Stress from uncertainty and resilience among depressed and burned out residents: a cross-sectional study. Acad Pediatr 18:698-704

9. Cooke GP, Doust JA, Steele MC (2013) A survey of resilience, burnout, and tolerance of uncertainty in Australian general practice registrars. BMC Med Educ 13:2

10. Allison JJ, Kiefe CI, Cook EF et al (1998) The association of physician attitudes about uncertainty and risk taking with resource use in a Medicare HMO. Med Decis Mak 18:320-329

11. Newman-Toker DE, McDonald KM, Meltzer DO (2013) How much diagnostic safety can we afford, and how should we decide? A health economics perspective. BMJ Qual Saf 22(Suppl 2):ii11-ii20

12. Strout TD, Hillen M, Gutheil C et al (2018) Tolerance of uncertainty: a systematic review of health and healthcare-related outcomes. Patient Educ Couns 101:1518-1537

13. King N (2018) Template analysis website: University of Huddersfield. https://research.hud.ac.uk/research-subjects/human-health/ template-analysis/ Accessed 1 Aug 2018

14. Paulus TM, Lester JN (2016) ATLAS.ti for conversation and discourse analysis studies. Int J Soc Res Method 19:405-428

15. Hillen MA, Gutheil CM, Strout TD et al (2017) Tolerance of uncertainty: conceptual analysis, integrative model, and implications for healthcare. Soc Sci Med 180:62-75

16. Peters S, Clarebout G, Diemers A et al (2017) Enhancing the connection between the classroom and the clinical workplace: a systematic review. Perspect Med Educ 6:148-157

17. Cristancho SM, Apramian T, Vanstone $M$ et al (2013) Understanding clinical uncertainty: what is going on when experienced surgeons are not sure what to do? Acad Med 88:1516

Publisher's Note Springer Nature remains neutral with regard to jurisdictional claims in published maps and institutional affiliations. 